\title{
Structural Causes of Right Bundle Branch Block-Time for a Closer Look?
}

\author{
James Ker* \\ Department of Physiology, University of Pretoria, Pretoria, South Africa, PO Box 24318, Gesina, Pretoria, South \\ Africa, 0031
}

\begin{abstract}
Right bundle branch block is an electrocardiographic phenomenon with specific criteria.
Currently, two specific forms of right bundle branch block are acknowledged, a proximal and a distal variant. A vast array of pathologies can cause proximal, distal or even combined forms of right bundle branch block.

In this study it is suggested that a third type of right bundle branch block exist: one caused by a subaortic muscular tendon in the left ventricle, leading to an increased velocity of conduction in the left ventricle, with a resultant "relative" right bundle branch block. It is concluded that it is necessary (and time) to take a closer look at endoventricular structures in the assessment of structural causes of right bundle branch block.
\end{abstract}

Keywords: Right bundle branch block, subaortic, tendon, structural.

\section{INTRODUCTION}

Right bundle branch block (RBBB) is an electrocardiographic phenomenon with specific criteria [1,2]: QRS duration > $120 \mathrm{msec}$; broad and/or notched R waves (rsr', rsR' or rSR pattern) in the right precordial leads (leads V1 and V2) and wide and/or deep S waves in leads I and V6.

RBBB is the electrocardiographic reflection of delayed conduction in the right ventricle, caused by sclerosis (Lenegre's disease), fibrosis (Lev`s disease) or necrosis of the right bundle branch [3, 4]. Recently, Shah et al. [5] described a case of a septal branch aneurysm which caused a right bundle branch block by causing direct pressure on the right bundle branch near it's subendocardial course on the right ventricular side of the interventricular septum.

It has been established that there are two forms of RBBB [6-8]: In the first, there is interruption of conduction in the main right branch of the bundle of His - termed proximal RBBB and in the second, conduction in the terminal ramifications of the right bundle branch are delayed - termed distal RBBB or arborisation block.

Interestingly, it has been shown that the clinical examination can guide the clinician to distinghuish between proximal and distal RBBB [6,9]. Brooks et al. [9] demonstrated echophonocardiographically that in patients with proximal RBBB the time interval between mitral valve and tricuspid valve closure was prolonged, whereas in patients with distal RBBB the time delay is between tricuspid valve closure and pulmonary valve opening.

In this study a third cause of RBBB is proposed - neither proximal, nor distal, but one caused by increased conduction

*Address correspondence to this author at the Department of Physiology, University of Pretoria, Pretoria, South Africa, PO Box 24318, Gesina, Pretoria, South Africa, 0031; Tel: +2712-3430078; Fax: +2712-3430079;

E-mail: jker@wol.co.za to the left ventricle by a subaortic muscular tendon in this way causing a "pseudo" RBBB.

\section{MATERIALS AND METHODS}

This was a prospective, observational study. A total of 880 patients who presented for a cardiovascular examination were examined for the presence of right bundle branch block (RBBB). 880 patients were screened. The number of patients amounted to 880 , due to the study protocol stipulating a time period of 6 months for enrollment.

All patients with RBBB with any history of structural heart disease and/or myocardial infarction were excluded from the study.

\section{RESULTS}

A total of 11 patients with RBBB were found. In this group of 11 patients 6 had a history of myocardial infarction and were excluded from the study. 2 of the remaining 5 had no detectable structural anomaly of the heart. However, in 3 of these 5 patients (Figs. 1, 2, 3) a peculiar muscular tendon extended between the subaortic portion of the interventricular septum and the left ventricular apex (Figs. 4, 5, 6).

\section{DISCUSSION}

Anatomically, the right bundle is composed of a single group of fibres which arborises only at the periphery [6]. Current physiological evidence demonstrates that the electrocardiographical pattern of RBBB is identical in both proximal and distal interruptions (block) of the right-sided His-Purkinje network [6, 10-12]. It has been shown that interruption of the proximal portion of the right-sided His-Purkinje network leads to a delay in the onset of right ventricular contraction (manifesting clinically as a delay between tricuspid and mitral valve closure) with a normal subsequent sequence of right ventricular contraction, 


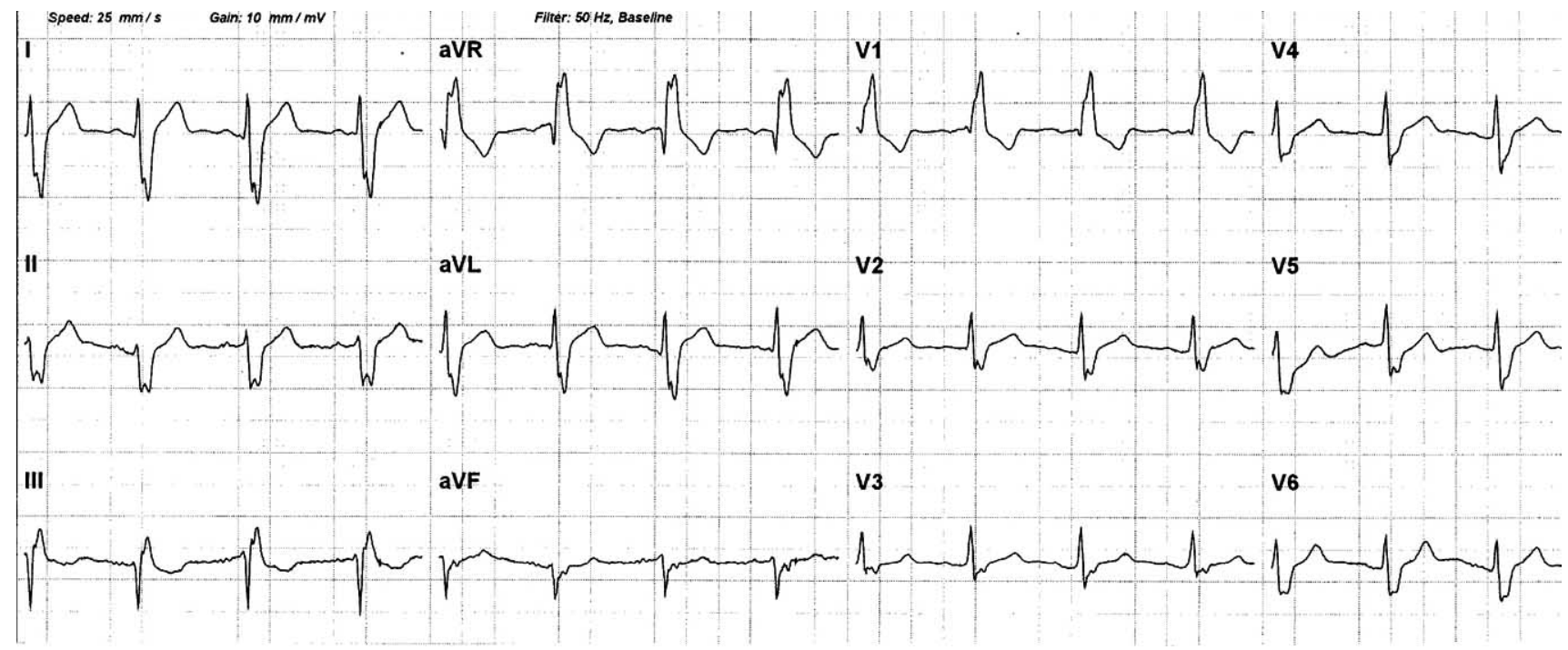

Fig. (1). The ECG clearly demonstrates a right bundle branch block.

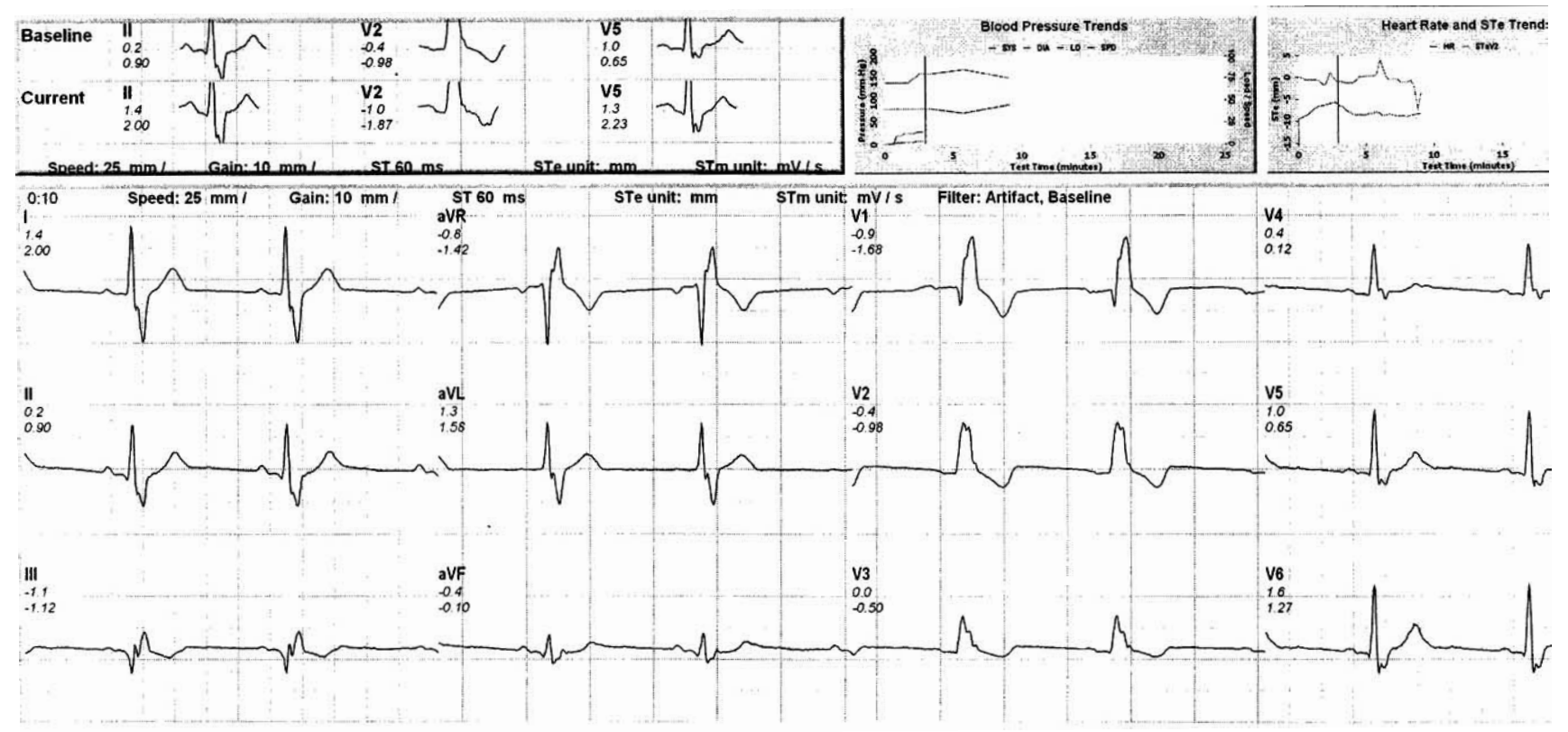

Fig. (2). Right bundle branch block is present.

whereas a distal block (disease affecting the distal branches of the His-Purkinje network) will cause asynchronous contraction of the right ventricle, thus slowing the rate of rise of pressure in the right ventricle, without delaying its onset, manifesting as a delay in the opening of the pulmonary valve[6, 10-12].

Combined disease is also possible in the same patient, with generalized disease affecting the right-sided conduction system, causing both proximal and distal RBBB [6].

Prognostically, it has been shown that this distinction is important, as proximal block caused by a single, localized lesion has an excellent long-term prognosis, whereas distal block caused by diffuse disease may be a manifestation of a progressive process $[6,13]$.
In this study, it is proposed that there exists a third type of right bundle branch block-neither proximal, nor distal, but one caused by an increased velocity of conduction to the left ventricle, effected by a muscular subaortic tendon coursing between the sub-aortic portion of the interventricular septum and the apex of the left ventricle, in this way leading to a "relative" $\mathrm{RBBB}$ as conduction in the right ventricle lags behind the increased conduction in the left ventricle.

Physiologically, this third type of RBBB behaves as a proximal block, with a delay in right ventricular contraction, manifested by a delay between mitral and tricuspid valve closure. This is not the first electrocardiographic 


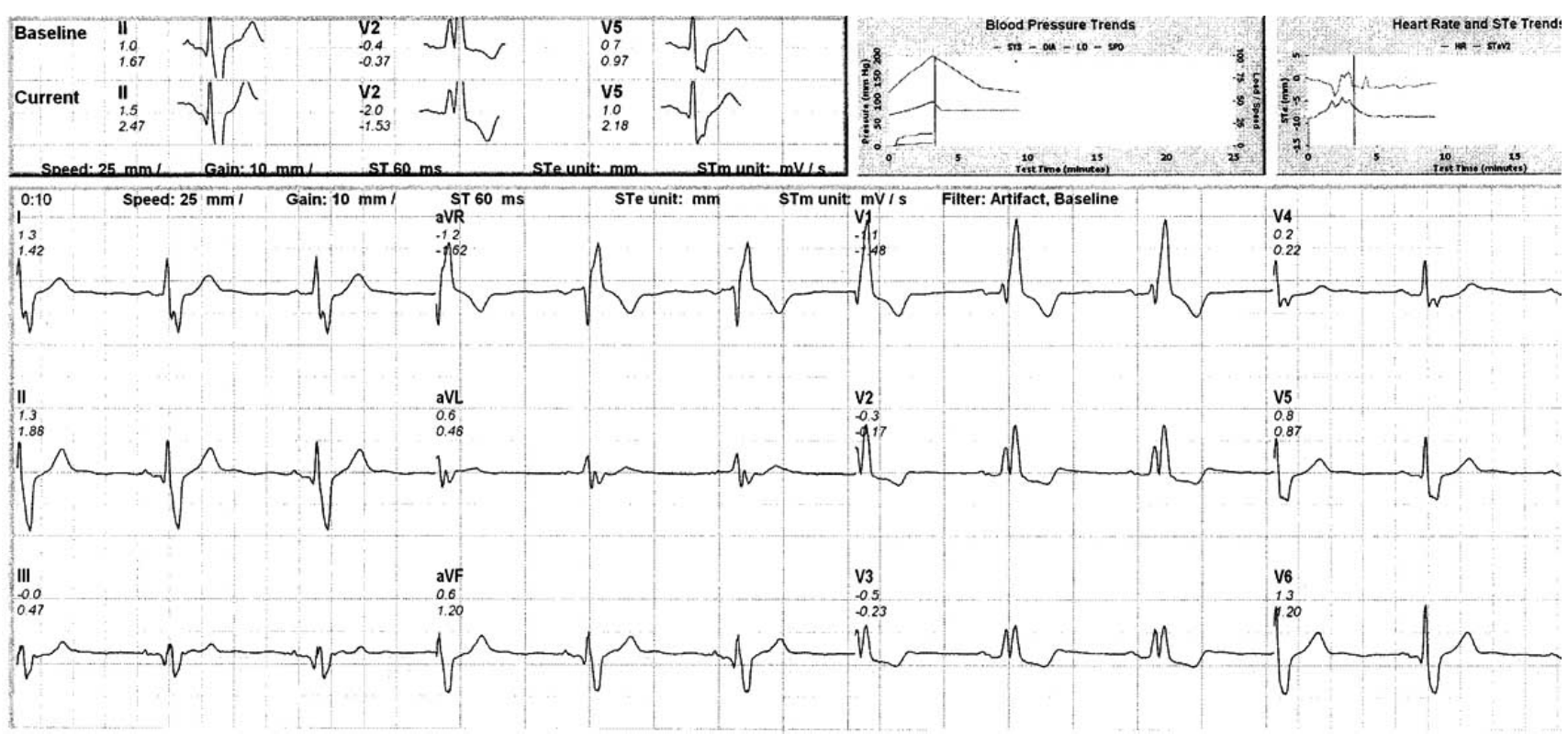

Fig. (3). Right bundle branch block is present.

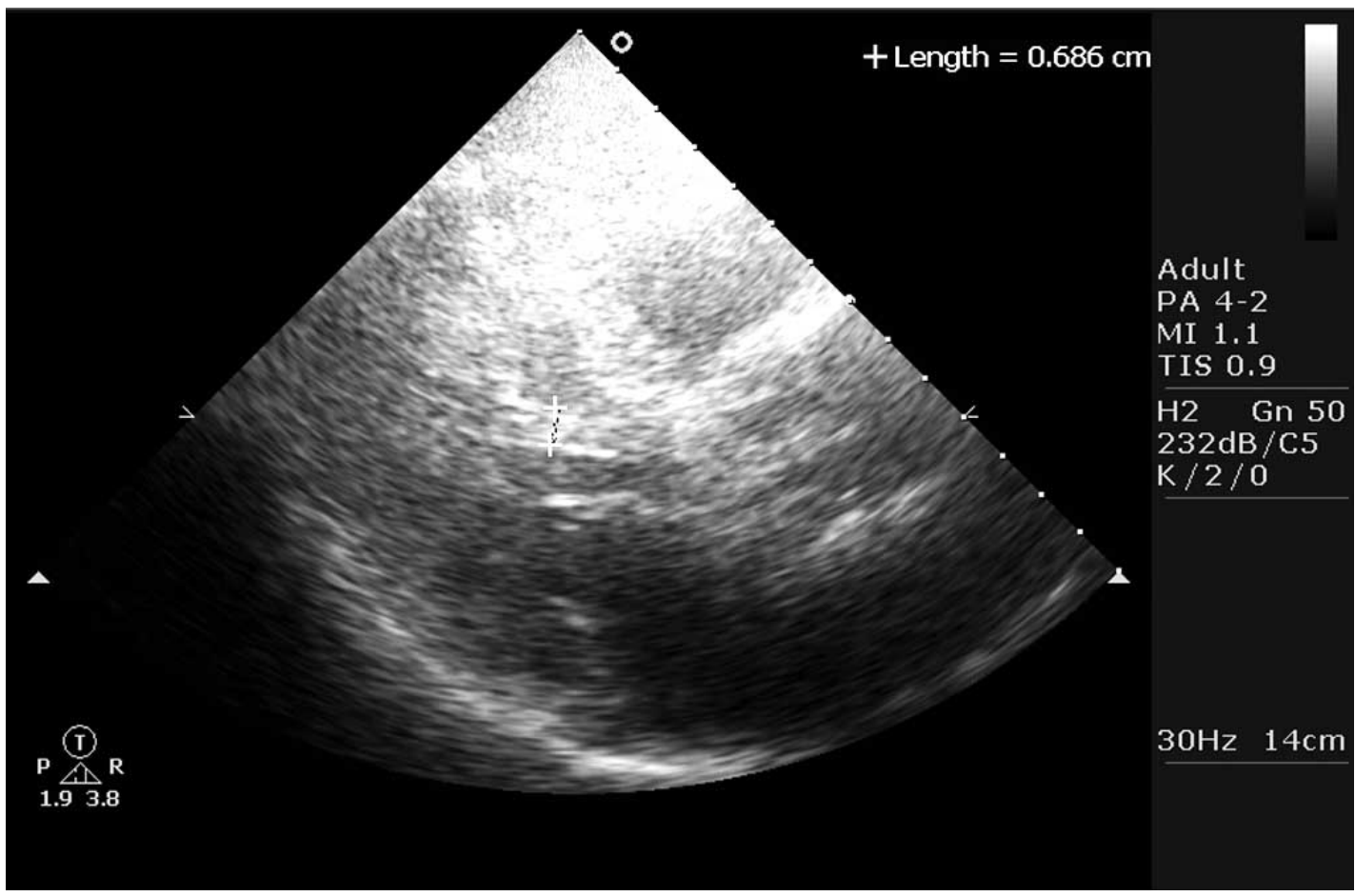

Fig. (4). Parasternal long axis view from patient 1, demonstrating the muscular subaortic tendon, arising from the subaortic area and implanting in the apex of the left ventricle.

manifestation of muscular, sub-aortic tendons: Ker [14] demonstrated that such a tendon can be responsible for striking ST-segment elevation on the electrocardiogram.

The electrocardiographic feature shared by all three these cases is a broad and notched R wave in lead aVR (Figs. 1, 2 and 3). Furthermore, they also share a deep and notched $S$ wave in leads I, II, aVL and V4, 5 and 6 with a broad R wave in lead V1.
The echocardiographic feature (Figs. 4, 5 and $\mathbf{6}$ ) is that of a thick and muscular band or tendon like structure extending from the subaortic area to the left ventricular apex. These muscular tendons are different from the widely known thin tendon like structures as they appear much thicker and they always extend from the subaortic region to the left ventricular apex. As stated before they have been shown to be a cause of ST segment elevation [14] and recently 


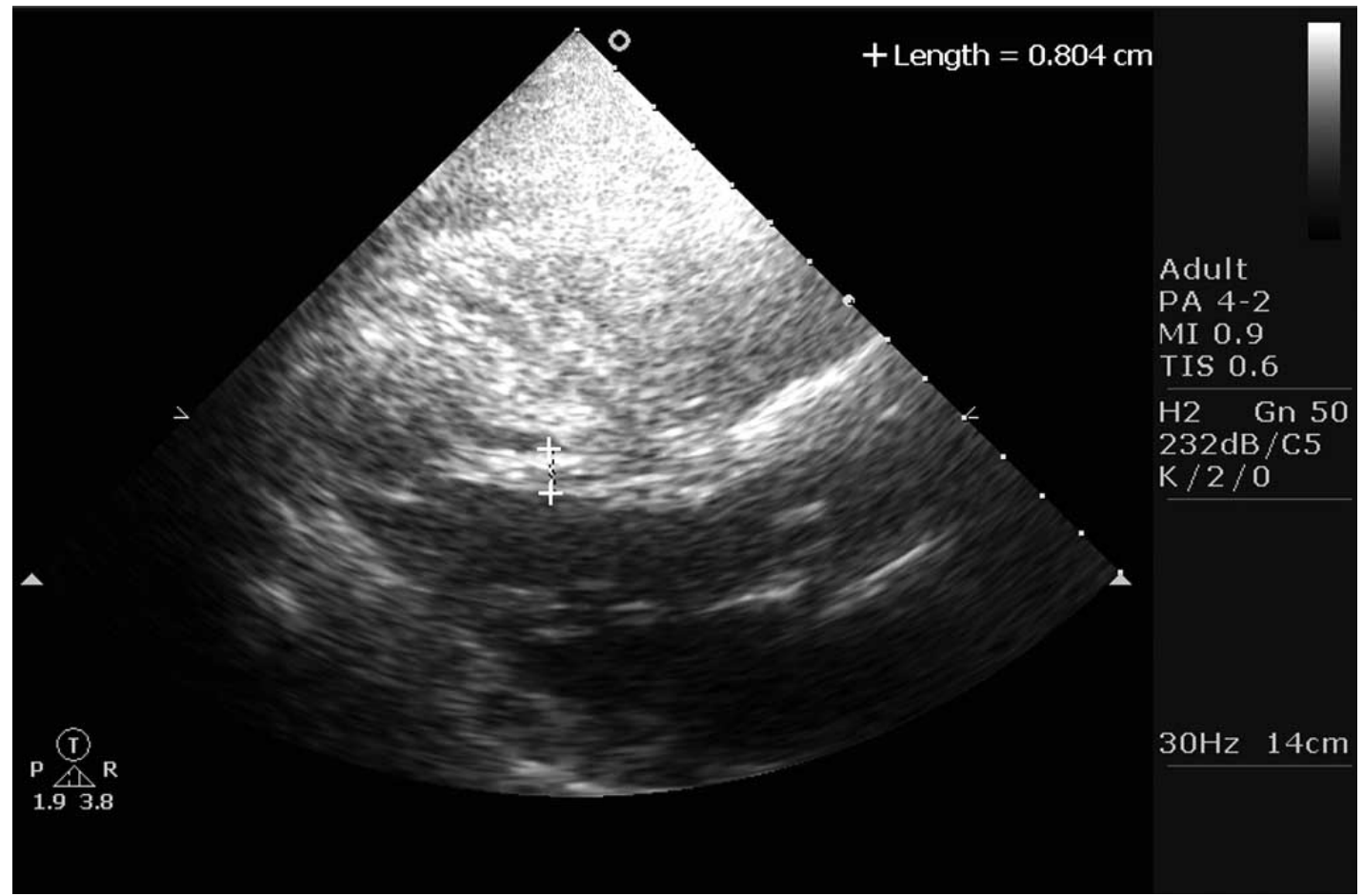

Fig. (5). Subaortic tendon in patient 2.

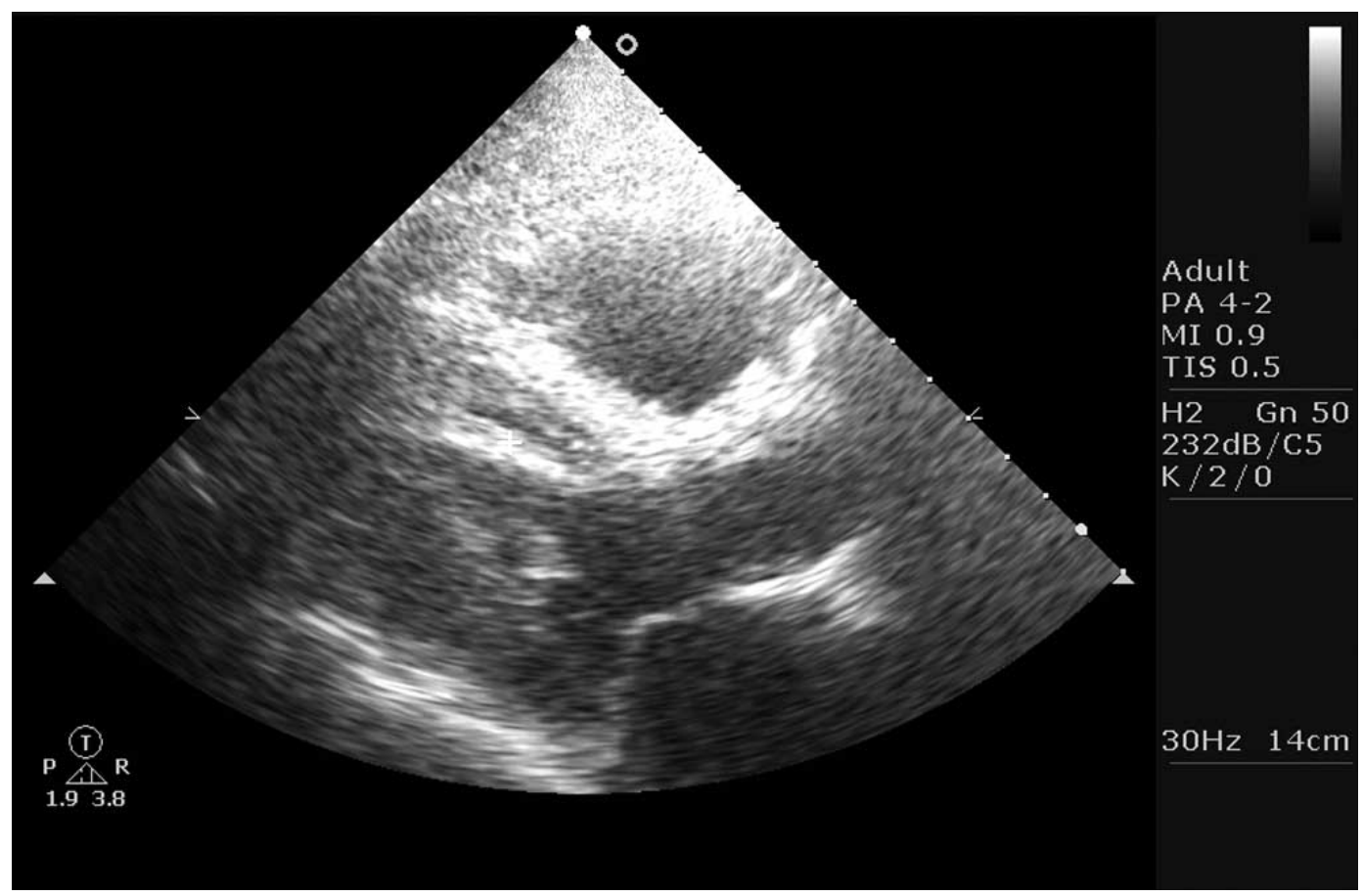

Fig. (6). Subaortic tendon in patient 3.

they have been shown to mimic hypertrophic cardiomyopathy [15].

Thus, in the assessment of structural causes for right bundle branch block it is suggested that it is time to take a closer look at endoventricular structures and specifically at muscular structures traversing the cavity of the left ventricle. Based on the demonstrated electrocardiograms it is suggested that a broad and notched $\mathrm{R}$ wave in lead aVR in patients with RBBB may serve as an electrocardiographic clue for an underlying endoventricular cause of RBBB.

\section{REFERENCES}

[1] Mirvis DM, Goldberger AL. Chapter 12: Electrocardiography. In: Braunwald's Heart Disease. A Textbook of Cardiovascular Medicine. $8^{\text {th }}$ ed, USA: Saunders Elsevier 2008. 
[2] Wagner GS. Chapter 5: Intraventricular conduction abnormalities. In: Marriott's Practical Electrocardiography. $9^{\text {th }}$ ed, London: Williams and Wilkins 1994

[3] Oscal A, Yildirim N, Ozbakir C, et al. Right bundle branch block: A new parameter revealing the progression rate of mitral stenosis. Cardiology 2006; 105: 219-22.

[4] Agarwal AK, Venugopalan P. Right bundle branch block: varying electrocardiographic patterns. Aetiological correlation, mechanisms and electrophysiology. Int J Cardiol 1999; 71: 33-9.

[5] Shah R, Shen M, Kirsch J. Septal branch aneurysm during coronary computed tomography angiography: a cause of right bundle branch block? Eur Heart J 2009; 30: 515

[6] Dancy M, Leech G, Leatham A. Significance of complete right bundle branch block when an isolated finding. An echocardiographic study. Br Heart J 1982; 48: 217-21.

[7] Rosenman RH, Pick A, Katz LN. The electrocardiographic patterns and the localization of intraventricular conduction defects. Am Heart J 1950; 40: 845-66.

[8] Braunwald E, Morrow AG. Sequence of ventricular contraction in human bundle branch block. Am J Med 1957; 23: 205-11.
[9] Brooks N, Leech G, Leatham A. Complete right bundle branch block. Echophonocardiographic study of first heart sound and right ventricular contraction times. Br Heart J 1979; 41: 637-46.

[10] Smith LA, Fields J, Kennamer R, Prinzmetal M. Studies on the mechanism of ventricular activity. III: Contraction of the ventricles in experimental bundle branch block. Am Heart J 1952; 44: 231-7.

[11] Abildskov JA, Eich RH, Harumi K, Smulyan H. Observations on the relation between ventricular activation sequence and the hemodynamic state. Circ Res 1965; 17: 236-47.

[12] Millar K, Burgess MJ, Abildskov JA, Eich RH. Dependence of intraventricular pressure on activation order before and after experimental Purkinje net block. J Electrocardiol 1970; 3: 516.

[13] Rotman M, Triebwasser JH. A clinical and follow-up study of right and left bundle branch block. Circulation 1975; 51: 477-84.

[14] Ker J. Sub aortic tendon induced ST segment elevation--a new echo electrocardiographic phenomenon? Cardiovasc Ultrasound 2009; 7: 13 .

[15] Ker J. The subaortic tendon as a mimic of hypertrophic cardiomyopathy. Cardiovas Ultrasound 2009; 7: 31.

(C) James Ker; Licensee Bentham Open.

This is an open access article licensed under the terms of the Creative Commons Attribution Non-Commercial License (http://creativecommons.org/licenses/ by-nc/3.0/) which permits unrestricted, non-commercial use, distribution and reproduction in any medium, provided the work is properly cited. 